\title{
Aprendizaje a distancia e integración tecnológica por la pandemia del Covid-19 en Perú
}

\section{Distance learning and technological integration due to the Covid- 19 pandemic in Peru}

Roberto Carlos Dávila Morán

rdavila430@gmail.com

https://orcid.org/0000-0003-3181-8801

Universidad Privada del Norte, Lima, Perú

Eucaris del Carmen Agüero Corzo

caricorzo@gmail.com

https://orcid.org/0000-0003-4587-3852

Universidad Pedagógica Experimental Libertador, Maturín, Venezuela

Recibido: 01/01/2021

Aceptado: 26/01/2021

\section{Resumen}

El objetivo del presente estudio consistió en examinar los problemas en particular con respecto a las tecnologías utilizadas, en el proceso de educación a distancia y los niveles de adopción de la educación a distancia de los docentes. La investigación se realizó bajo el enfoque cuantitativo de tipo descriptivo, con una muestra constituida por 572 maestros en Perú, en el que se aplicó un cuestionario de Google Forms en línea. Los resultados revelan que los profesores se encuentran en un nivel de mejora de la integración de la tecnología y, por lo tanto, integran la tecnología como una transformación del aprendizaje.

Palabras clave: Integración de la tecnología de los docentes, adopción de la educación a distancia, educación a distancia, COVID-19.

\section{Abstract}

The objective of the present study was to examine the problems in particular with respect to the technologies used in the distance education process and the levels of adoption of distance education by teachers. The research was carried out under a descriptive quantitative approach, with a sample consisting of 572 teachers in Peru, in which an online Google Forms questionnaire was applied. The results reveal that teachers are at a level of improvement of the integration of technology and, therefore, they integrate technology as a transformation of learning. 
Keywords: Teacher's technology integration, distance learning adoption, distance learning, COVID-19.

\section{Introducción}

De repente, profesores y estudiantes de todo el mundo tienen que lidiar con la enseñanza y el aprendizaje a distancia durante la crisis de la enfermedad del COVID19 como consecuencia de una política de trabajo desde casa para prevenir una mayor transmisión del virus. De hecho, el brote está causando varios niveles de interrupciones en la educación a nivel mundial iniciados con cierres de escuelas en 165 países que impactan a más de 1.500 millones de estudiantes (Doucet et al., 2020). Una variedad de enfoques y estrategias de aprendizaje intentados por los profesores con el fin de ejecutar el aprendizaje remoto sin problemas para que los estudiantes pudieran aprender tan conveniente como en el aprendizaje cara a cara. No hay preparación formal de antemano para enfrentar este desafío; en consecuencia, los profesores estaban lidiando con esta situación por su propia creatividad. Los estudiantes se mantuvieron alejados de las escuelas y se metieron en una circunstancia diferente que la que han conocido. Además, era inevitable que los padres se involucraran en los procesos de aprendizaje para educar a sus hijos porque están estudiando desde casa. La enseñanza en un momento tumultuoso, requiere una alta sensación de innovación, así como una generosa flexibilidad para promover en lugar de actividades de aprendizaje lamentables (Martinez-Cola et al., 2018).

En medio del impacto mundial sin precedentes del COVID-19, particularmente hacia el sector de la educación, es cada vez más importante entender la integración tecnológica de los profesores y la adopción del aprendizaje a distancia después de una implementación repentina de la política de trabajo desde casa. El término integración de tecnología significa el uso efectivo de las tecnologías de la información y la comunicación (Tondeur et al., 2009) y la implementación apropiada de tecnologías educativas para lograr los resultados de aprendizaje previstos (Davies y West, 2014). La adopción se trata de la elección individual de aceptar o rechazar innovaciones particulares integradas en un contexto apropiado (Hall y Khan, 2003; Straub, 2009). Además, la adopción de tecnología se refiere a una consideración individual para tomar una decisión de aceptar y utilizar nuevas tecnologías o incluso uno rechazarla (Sepasgozar y Davis, 2018). En términos generales, parece que los profesores utilizan una variedad de tecnologías para llevar a cabo un aprendizaje remoto asincrónico o sincrónico desde casa, mientras hay una agilidad distintiva en la adopción del aprendizaje a distancia a su alcance. No obstante, los detalles exactos sobre las tecnologías integradas y los niveles de adopción en el meollo del brote de COVID-19 siguen siendo un problema. 


\section{Metodología}

El estudio de la tecnología de los profesores y la adopción del aprendizaje a distancia durante la crisis del COVID-19 es una investigación transversal descriptiva realizada en Perú mediante el uso de una encuesta en línea. El diseño de la investigación fue elegido debido a su efectividad para recopilar datos y estudiar múltiples resultados en un solo momento (Levin, 2006; Wang y Cheng, 2020). Tras el diseño de la investigación, la encuesta se administró dos semanas después de que el Gobierno implementa una política de trabajo desde casa para todos los empleados, incluidos profesores, a partir del 16 al 27 de marzo de 2020. En esta situación crítica, los profesores deben enseñar a los estudiantes a distancia desde casa sin cualquier preparación formal.

Se desarrolló un cuestionario en Google Forms y se distribuyó a los profesores mediante una muestra de conveniencia a través de una variedad de redes sociales como WhatsApp, Facebook y Twitter dentro de los cinco días hábiles entre el 30 de marzo y el 3 de abril de 2020. El cuestionario, en particular sobre el proceso de aprendizaje a distancia, fue desarrollado en base a la versión revisada del dominio cognitivo de la taxonomía de Bloom (Anderson y Bloom, 2001), aprendiendo teorías del conductismo, cognitivismo, constructivismo, conectivismo, y la clasificación de recursos de aprendizaje. Además, la reflexión de los profesores sobre el nivel de adopción del aprendizaje a distancia se adoptó de la curva de adopción de innovaciones de Rogers (1962).

En cuanto a la técnica de análisis de datos, se ha utilizado una estadística descriptiva para simplificar, analizar y describir las principales características de los datos recopilados, así como visualizar los datos en gráficos muy comprensibles (Holcomb, 2016). En lo que respecta al estudio ético, se indicó explícitamente de antemano que todos los datos recopilados se utilizan únicamente con fines de investigación.

Aparte de que la política se prolonga hasta el final del semestre escolar, este estudio retrata una experiencia temprana de profesores enseñando a sus estudiantes en las dos primeras semanas de la política de trabajo desde casa en Perú.

\section{Resultados y discusiones}

Después de la difusión de una semana, 572 profesores completaron el cuestionario en línea. En la tabla $\mathrm{N}^{\circ} 1$ está la información detallada sobre el perfil de los profesores con respecto a su género, edad, nivel educativo, experiencia docente, sistema escolar, tipo de escuela y ubicación. 
Tabla $\mathbf{N}^{\circ} \mathbf{1}$. Perfil de los profesores en el estudio.

\begin{tabular}{|c|c|c|c|c|c|}
\hline Genero & $\begin{array}{c}\text { Hombre } \\
182 \\
31.82 \%\end{array}$ & $\begin{array}{c}\text { Mujer } \\
390 \\
68.18 \%\end{array}$ & & & \\
\hline Edad & $\begin{array}{c}<25 \\
68 \\
11.89 \%\end{array}$ & $\begin{array}{c}25-35 \\
260 \\
45.45 \%\end{array}$ & $\begin{array}{c}36-45 \\
124 \\
21.68 \%\end{array}$ & $\begin{array}{c}46-55 \\
101 \\
17.66 \%\end{array}$ & $\begin{array}{c}>55 \\
19 \\
3.32 \%\end{array}$ \\
\hline Nivel educativo & $\begin{array}{c}\text { Asociado } \\
8 \\
1.40 \%\end{array}$ & $\begin{array}{c}\text { Bachiller } \\
471 \\
82.34 \%\end{array}$ & $\begin{array}{c}\text { Maestría } \\
87 \\
15.21 \%\end{array}$ & $\begin{array}{c}\text { Doctor } \\
6 \\
1.05 \%\end{array}$ & \\
\hline Experiencia docente & $\begin{array}{c}<5 \\
185 \\
32.34 \%\end{array}$ & $\begin{array}{c}5-10 \\
154 \\
26.92 \%\end{array}$ & $\begin{array}{c}11-15 \\
111 \\
19.41 \%\end{array}$ & $\begin{array}{c}16-20 \\
46 \\
8.04 \%\end{array}$ & $\begin{array}{c}>20 \\
76 \\
13.29 \%\end{array}$ \\
\hline Sistema escolar & $\begin{array}{c}\text { Primaria } \\
289 \\
50.52 \%\end{array}$ & $\begin{array}{c}\text { Secundaria } \\
283 \\
49.48 \%\end{array}$ & & & \\
\hline Tipo de escuela & $\begin{array}{c}\text { Privado } \\
191 \\
33.39 \%\end{array}$ & $\begin{array}{c}\text { Publico } \\
381 \\
66.61 \%\end{array}$ & & & \\
\hline Ubicación & $\begin{array}{c}\text { Piura } \\
137 \\
23.95 \%\end{array}$ & $\begin{array}{c}\text { Lima } \\
323 \\
56.47 \%\end{array}$ & $\begin{array}{c}\text { Moquegua } \\
33 \\
5.77 \%\end{array}$ & $\begin{array}{c}\text { Tumbes } \\
45 \\
7.87 \%\end{array}$ & $\begin{array}{c}\text { Otros } \\
34 \\
5.94 \%\end{array}$ \\
\hline
\end{tabular}

La tabla $\mathrm{N}^{\circ} 1$ describe los perfiles de los profesores que participaron en este estudio. Casi el 70\% de los participantes son mujeres. La mayoría de los profesores de entre 25 y 35 años alcanzan más del $45 \%$ mientras que los docentes de más de 55 años son minoría. Además, la gran mayoría de los profesores tiene una licenciatura logrando más del $80 \%$. Sin embargo, más de la mitad de los profesores tienen experiencia en enseñar menos de 10 años. Alrededor del 49\% de los profesores enseñan en secundaria, mientras tanto, el número de profesores que enseñan en primaria es alrededor del $51 \%$. En cuanto al tipo de escuela, alrededor de dos tercios de los profesores trabajan en colegios públicos mientras que el resto trabaja en colegios privados. Además, más del $50 \%$ de los profesores viven en Lima, el $24 \%$ de los profesores viven en Piura y un pequeño número de profesores de Moquegua y Tumbes, y otros departamentos.

Tecnologías utilizadas por los profesores para la enseñanza a distancia

Los profesores utilizan amplias tecnologías para llevar a cabo el aprendizaje a distancia (ver tabla $\mathrm{N}^{\circ} 2$ ). Los dispositivos incluyen una computadora de escritorio, laptops, Smartphone y Tablet. 
Roberto Carlos Dávila Morán, Eucaris del Carmen Agüero Corzo.

Tabla N². Tecnologías utilizadas por los profesores para la enseñanza a distancia.

\begin{tabular}{|c|c|c|c|c|}
\hline \multicolumn{5}{|c|}{ Dispositivos } \\
\hline $\begin{array}{l}\text { Computadoras } \\
\text { de escritorio } \\
43 \\
7.52 \%\end{array}$ & $\begin{array}{c}\text { Smartphones } \\
319 \\
55.77 \%\end{array}$ & $\begin{array}{c}\text { Laptops } \\
170 \\
29.72 \%\end{array}$ & $\begin{array}{c}\text { Tablets } \\
40 \\
6.99 \%\end{array}$ & \\
\hline \multicolumn{5}{|c|}{ Lecciones sincrónicas } \\
\hline $\begin{array}{l}\text { Google meet } \\
54 \\
9.44 \%\end{array}$ & $\begin{array}{c}\text { WhatsApp VC } \\
323 \\
56.47 \%\end{array}$ & $\begin{array}{c}\text { Zoom } \\
121 \\
21.15 \%\end{array}$ & $\begin{array}{c}\text { YouTube live } \\
38 \\
6.64 \%\end{array}$ & $\begin{array}{c}\text { Facebook } \\
\text { Live } \\
36 \\
6.29 \%\end{array}$ \\
\hline \multicolumn{5}{|c|}{ Comunicación con los estudiantes } \\
\hline $\begin{array}{l}\text { WhatsApp } \\
540 \\
94.41 \%\end{array}$ & $\begin{array}{c}\text { Telegram } \\
13 \\
2.27 \%\end{array}$ & $\begin{array}{c}\text { Line } \\
9 \\
1.57 \%\end{array}$ & $\begin{array}{c}\text { Twitter } \\
4 \\
0.70 \%\end{array}$ & $\begin{array}{c}\text { WeChat } \\
6 \\
1.05 \%\end{array}$ \\
\hline \multicolumn{5}{|c|}{ Aula virtual } \\
\hline $\begin{array}{l}\text { Google } \\
\text { Classroom } \\
345 \\
60.31 \%\end{array}$ & $\begin{array}{l}\text { Brainly } \\
53 \\
9.27 \%\end{array}$ & $\begin{array}{c}\text { Edmodo } \\
48 \\
8.39 \%\end{array}$ & $\begin{array}{c}\text { Office } 365 \\
85 \\
14.86 \%\end{array}$ & $\begin{array}{c}\text { Quipper } \\
41 \\
7.17 \%\end{array}$ \\
\hline
\end{tabular}

La tabla $\mathrm{N}^{\circ} 2$ resume diversas tecnologías utilizadas por los profesores como herramientas pedagógicas para la realización de aprendizaje a distancia. En general, hay algunos dispositivos y aplicaciones que se usan comúnmente para facilitar el aprendizaje a los estudiantes remotamente. Los Smartphones y las laptops son los dispositivos más populares preferidos con un $55.77 \%$ y $29.72 \%$ de los profesores respectivamente. Para la enseñanza en vivo en línea, más del $56 \%$ de los profesores utilizan videollamada de WhatsApp y zoom fue utilizado por alrededor del $21 \%$ de los profesores. Mientras que otras aplicaciones fueron utilizadas por menos del $10 \%$ de profesores. En cuanto a las aplicaciones para comunicarse con estudiantes, WhatsApp es el más popular utilizado por casi el $100 \%$ de los profesores, mientras que, en contraste, Telegram, Line, Twitter, y WeChat son de uso poco común por los profesores. Por último, Google Classroom y Microsoft Office 365 son las aplicaciones más populares para realizar una lección en el entorno virtual con un $60.31 \%$ y $14.86 \%$ respectivamente.

No es de extrañar que la gran mayoría de los profesores aprovechen los Smartphones para realizar sesiones a distancia, ya que los Smartphones son el dispositivo móvil más utilizado en Perú. Aunque aprovechar los Smartphones para el aprendizaje remoto en el contexto de la educación superior también es relativamente una práctica nueva, se ha demostrado que los usos mejoran los compromisos de aprendizaje de los estudiantes y facilitan la comunicación entre estudiantes y profesores (Vázquez-Cano, 2014; Tuncay, 2016). Los Smartphones apoyan el aprendizaje a distancia en tiempo real y la colaboración sincrónica entre los estudiantes y profesores (Lee, 2012). Por lo tanto, los Smartphones como herramientas pedagógicas inteligentes promueven el

102 Aprendizaje a distancia e integración tecnológica por la pandemia del Covid-19 en Perú. - Eduweb, 2021, enero-abril, v.15, n.1. / 98-111 
aprendizaje ubicuo (Shin et al., 2011). Maximizar el uso de Smartphones en este caso podría ser también una solución del aprendizaje a distancia tradicional basado en computadoras que no son totalmente adecuadas debido a la falta de laptops y computadores de escritorio.

Curiosamente, la aplicación WhatsApp es utilizada por más de la mitad de los profesores para enseñanzas sincrónicas, mientras que las otras aplicaciones son menos populares con excepción de Zoom. Además, WhatsApp es también el principal medio para la comunicación a distancia entre profesores y estudiantes. Este hallazgo revela que WhatsApp facilita la enseñanza y el aprendizaje a distancia. Razones por las que WhatsApp es altamente aceptable para el aprendizaje a distancia debido a la gran facilidad de uso, accesibilidad y rentabilidad (Nawaila y Bicen, 2018; Singh et al., 2018). Algunos estudios han confirmado la potencialidad de diferentes formas en función de las características como el chat de WhatsApp (Cetinkaya y Sütçü, 2018; Saleh, 2019) y grupo de WhatsApp (Annamalai, 2019) o apoyando actividades efectivas de aprendizaje e instrucción. Zoom y las otras herramientas de conferencia recientemente ganan cada vez más popularidad en medio del brote de COVID-19 como medio para tener aulas en línea sincrónicas. Otro punto importante a tener en cuenta es que los profesores también han intentado utilizar varias redes sociales para el aprendizaje a distancia. Esta nueva forma de enseñar es un gran desafío tanto para profesores como estudiantes que requieren diferentes estrategias de enseñanza y aprendizaje en comparación con las instrucciones presenciales en vivo (Safei et al., 2011).

Los profesores utilizan una variedad de aplicaciones para gestionar entornos de aulas virtuales, la mayoría utiliza Google Classroom porque es un sistema de gestión de aprendizaje gratuito y fácil de usar (Heggart y Yoo, 2018; Rozak \& Albantani, 2018). Otras plataformas de aulas virtuales son menos populares, mientras que algunos profesores desarrollan y utilizan su propia aplicación. El desarrollo de una plataforma propia es posible mediante el uso de sistemas de gestión de aprendizaje como Moodle, Chamilo y Blackboard; sin embargo, parece más complicado en lugar de simplemente adoptar Google Classroom. La integración de un sistema de gestión del aprendizaje en las prácticas docentes requiere no solo conocimientos técnicos específicos, sino también nuevos conocimientos pedagógicos (Anderson y Dron, 2017; Adnan et al., 2017; Ouadoud et al., 2017). La naturaleza pedagógica y los problemas técnicos podrían disminuir la intención de los profesores de aprovechar la oportunidad de utilizar un sistema de gestión para gestionar el aprendizaje virtual (Walker et al., 2016). Por lo tanto, el desarrollo de los conocimientos tecnológicos y pedagógicos específicos de los profesores es de importancia antes de integrar ciertas tecnologías para el aprendizaje. Reflexionando sobre las diversas tecnologías elegidas por los profesores, parece que los profesores tienden a utilizar tecnologías para la enseñanza remota que ya utilizaban para la vida diaria. Por ejemplo, los Smartphones y la aplicación de WhatsApp que ha sido ampliamente utilizada en las actividades diarias también son utilizados por los 
profesores para la enseñanza síncrona y para comunicarse con los estudiantes desde sus hogares.

El proceso de aprendizaje a distancia conducido por profesores

Las dificultades descritas en los procesos de aprendizaje a distancia incluyen actividades de aprendizaje, tareas y recursos que fueron dados a los estudiantes y utilizados por los profesores como un medio de enseñanza a distancia. Las actividades de aprendizaje fueron categorizadas sobre la base de la versión revisada del dominio cognitivo de la taxonomía de Bloom que incluye recordar, entender, aplicar, analizar, evaluar y crear (Anderson y Bloom, 2001). Además, aprender teorías del conductismo, cognitivismo, constructivismo y conectivismo aplicadas para clasificar los tipos de tareas. Para ser más detallados, las tareas y teorías de aprendizaje relacionadas incluyen: trabajar en pruebas de selección múltiple y de redacción asignados y recopilados a los profesores (conductismo). Leer libros de texto y luego escribir un resumen (cognitivismo), discutiendo materiales de aprendizaje con compañeros 0 padres y luego concluir la discusión escribiendo un resumen o dibujar una imagen como mapas conceptuales e infografías (constructivismo) y crear un producto en particular a partir del material de aprendizaje y luego compartirlo con compañeros u otras personas a través de diversos medios digitales (conectivismo). Varios recursos de aprendizaje utilizados durante el aprendizaje remoto, como libros de texto, entornos, compañeros, padres y recursos en línea, también se describen en la tabla Nº3.

Tabla $\mathbf{N}^{\circ}$ 3. El proceso de aprendizaje a distancia realizado por los profesores.

\begin{tabular}{lccccl}
\hline \multicolumn{5}{c}{ Actividades } \\
\hline Recordar & Entender & Aplicar & Analizar & Evaluar & Crear \\
$\mathbf{1 0 9}$ & 455 & 233 & 304 & 285 & 307 \\
$\mathbf{1 9 . 0 6 \%}$ & $79.55 \%$ & $40.73 \%$ & $53.15 \%$ & $49.83 \%$ & $53.67 \%$ \\
\hline \multicolumn{5}{c}{ Tareas } \\
\hline Haciendo & Lectura de & Discutiendo & Creando \\
tarea & libros & materiales & productos \\
$\mathbf{4 7 0}$ & 286 & 162 & 234 \\
$\mathbf{8 2 . 1 7 \%}$ & $50 \%$ & $28.32 \%$ & $40.91 \%$ & \\
\hline \multicolumn{5}{c}{ Recursos } \\
\hline Libros de & Entornos & Compañeros & Padres & Recursos en \\
texto & 263 & 169 & 267 & Internet \\
$\mathbf{4 7 6}$ & $45.98 \%$ & $29.55 \%$ & $46.68 \%$ & 464 \\
$\mathbf{8 3 . 2 2} \%$ & \multicolumn{5}{c}{$81.12 \%$} \\
\hline
\end{tabular}

La tabla $\mathrm{N}^{\circ} 3$ revela los procesos de aprendizaje a distancia que realizan los profesores a sus estudiantes. En general, hay actividades, tareas y recursos típicos que se utilizan con frecuencia para aprender a distancia. Casi el $80 \%$ de las actividades se trata de comprender y es cuatro veces superior a los de recordar. Sorprendentemente, las actividades de analizar y crear algo a partir del material de aprendizaje tienen un 
número ligeramente compartido de alrededor del $53 \%$, y las otras dos actividades se registran menos del $50 \%$. De estos datos se desprende claramente que la gran mayoría de los profesores dan una tarea a los estudiantes por realizar, alcanzando más del $80 \%$. En cuanto a los recursos de aprendizaje, libros de texto y recursos en Internet son los recursos más frecuentes utilizados por los profesores que llegan a más del $80 \%$, alrededor del $45 \%$ de los profesores aprovechan entornos y padres como recursos para el aprendizaje, mientras que los compañeros solo fueron utilizados por alrededor del $30 \%$ de los profesores.

Entender el material de aprendizaje, como las principales actividades de aprendizaje de los estudiantes facilitados por sus profesores desde la distancia, implica que el proceso de aprendizaje se ejecuta en las habilidades de pensamiento de orden inferior. Este es un problema general en los procesos de enseñanza y aprendizaje de Perú independientemente del modo de aprendizaje presencial o a distancia. Se asume el conocimiento previo y las creencias de los profesores sobre las habilidades de pensamiento de orden superior como factores principales que inciden en el nivel de las prácticas pedagógicas en el aula. Aunque alrededor de la mitad de los profesores han realizado actividades basadas en habilidades de pensamiento de orden superior, es fundamental alentar a todos los profesores a avanzar desde los de nivel inferior.

Como hacer las tareas es un trabajo común del estudiante diario, parece que la mayoría de los profesores están enseñando remotamente de una manera conductista. Es más probable que los profesores den asignaciones basadas en pruebas en lugar de las asignaciones basadas en actividades, que pueden estimular a los estudiantes a pensar, construir o crear un determinado producto de los materiales de aprendizaje. Es cierto que la tarea juega un papel importante para el refuerzo del aprendizaje y para el desarrollo de la responsabilidad personal (Xu y Yuan, 2003; Corno y Xu, 2004); sin embargo, el uso de la tarea como trabajo principal de aprendizaje a distancia podría ser estresante, no sólo para los estudiantes, sino también para los padres durante el brote de COVID-19 (Suldo et al., 2008; Xu, 2011; Clausen et al., 2020). Mirar la tarea de manera diferente podría ser una solución al involucrar activamente aspectos culturales que aumentan el sentido de comunidad entre estudiantes, compañeros, padres y profesores en sus entornos sociales (Corno, 2000). Por lo tanto, por ejemplo, la construcción y discusión de material de aprendizaje con compañeros o padres, así como la creación y el intercambio de un producto a los llamamientos de la comunidad como tareas más fructíferas durante el aprendizaje a distancia.

La mayoría de los profesores confían en los libros de texto y otros recursos en Internet como recursos de aprendizaje a distancia en línea con el hecho de que la mayoría de las tareas de aprendizaje a distancia tienen más que ver con hacer los deberes en lugar de asignar otras tareas relacionadas con sus compañeros, padres y entornos a su alrededor. Del mismo modo, este es también un caso común en las prácticas de enseñanza cara a cara de Perú, que los profesores utilizan principalmente libros de texto como los principales recursos de aprendizaje. Los libros de texto han sido 
ampliamente utilizados para la enseñanza en la primera década de la educación y son realmente muy importantes para los recursos básicos de aprendizaje (Davey, 1988). Hoy en día, el uso más apropiado de libros de texto impresos debería combinarse o integrarse con otros recursos electrónicos o digitales (McDonald, 2016). En el momento del distanciamiento social y físico, fomentar la interacción activa entre estudiantes, padres y sus entornos de aprendizaje es valioso para el recurso de aprendizaje. Adicionalmente, tener una reunión virtual con compañeros puede ser beneficioso para apoyarse mutuamente sobre el progreso de su estudio.

Reflexionar sobre lo que han hecho los profesores con los procesos de educación a distancia facilitados por diversas tecnologías abre la posibilidad de predecir sus niveles de integración tecnológica. Se puede decir que los profesores se encuentran en un nivel de mejora de la integración de la tecnología, por lo que integran la tecnología como una transformación del aprendizaje. Basado en el modelo SAMR de integración tecnológica, los profesores integran la tecnología principalmente para sustituir y aumentar un proceso de aprendizaje a distancia de modificar y redefinir el aprendizaje. En otras palabras, los profesores siguen incursionando y haciendo cosas viejas a la antigua, aunque múltiples tecnologías inteligentes ya están en sus manos.

Además, desde la perspectiva de TPACK (Koehler y Mishra, 2005; Mishra y Koehler, 2006), los profesores aún no integran la tecnología en relación activa a la pedagogía y al contenido. Sin embargo, la experiencia al iniciar la integración de la tecnología para mejorar el aprendizaje podría ser valioso para avanzar hacia la etapa de transformación.

Niveles de adopción del aprendizaje a distancia de los profesores

Los niveles de adopción del aprendizaje a distancia de los profesores abordan varios niveles de profesores en la adopción del aprendizaje remoto en sus manos después de una implementación repentina de la política de trabajo desde casa. Adoptado de la curva de adopción de innovaciones de Rogers (1962), hay cinco niveles de adopción utilizados en este estudio y descritos a continuación: 1) innovadores = los profesores están preparados para el aprendizaje a distancia antes de la implementación de la política de trabajo desde casa; 2) primeros adoptantes = los profesores se preparan inmediatamente para el aprendizaje a distancia cuando la política de trabajo desde casa ha sido implementada; 3) mayoría temprana = los profesores comienzan a prepararse para el aprendizaje a distancia cuando la política de trabajar desde casa se ha implementado porque ver a otros maestros se estaban preparando para ella; 4) mayoría tardía = los profesores comienzan a prepararse para el aprendizaje a distancia cuando se ha aplicado la política de trabajo desde casa; 5) rezagados = profesores que no se preparan para el aprendizaje a distancia, aunque se haya implementado la política de trabajar desde casa, y piensan en prepararse más tarde. Los niveles de adopción del aprendizaje a distancia por parte de los profesores se ilustran en la figura $N^{\circ} 1$. 
Curva de adopción de innovación de Rogers

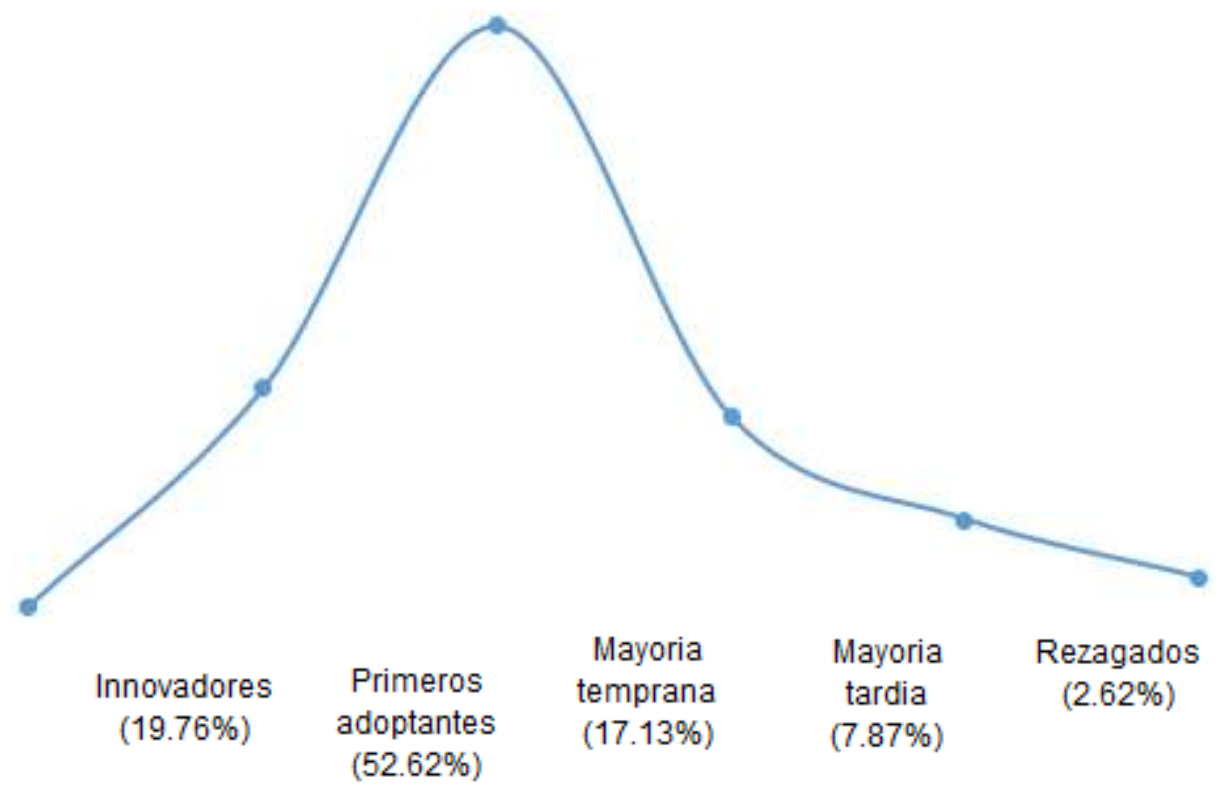

Figura №1. Curva de innovación de Rogers.

La figura $N^{\circ} 1$ ilustra los niveles de adopción de la educación a distancia de los profesores. En general, la mayoría de profesores definieron ellos mismos como los primeros en adoptar el aprendizaje a distancia. Más de la mitad de los profesores entran en esta categoría, mientras que la cifra para la mayoría temprana y tardía alcanzó el $17,13 \%$ y el $7,87 \%$ respectivamente. El número de innovadores es casi el $20 \%$ y la cifra de rezagados menos del $3 \%$. La curva es bastante diferente de la teoría del origen ya que conceptualmente debería haber un 2,5\% de innovadores, un $13,5 \%$ de adoptadores temprano, un 34\% de mayoría temprana, el 34\% de mayoría tardía y el $16 \%$ de rezagados (Rogers, 1962).

El nivel de adopción de los profesores en este estudio es consistente con el estudio de Aldunate y Nussbaum (2013) donde se revelo que la mayoría de los profesores en su estudio sobre la adopción de la tecnología por parte de los profesores caen en la categoría de pioneros y seguidos por innovadores. Los profesores que son los primeros en adoptar la tecnología tienen un tiempo en la incorporación de tecnología educativa y es más probable que adopten nuevas tecnologías a sus prácticas de enseñanza. Otro estudio, particularmente sobre la adopción de tecnología para el aprendizaje, también muestra que los profesores han adoptado sustancialmente la tecnología a un nivel moderado (Kotrlik y Redmann, 2009; Redmann y Kotrlik, 2009). Sin embargo, la tecnología no se ha integrado adecuadamente. Esto suena interesante ya que adoptar la tecnología no significa automáticamente que los profesores hayan utilizado la tecnología en medios adecuados para la enseñanza y el aprendizaje. 
Al reflexionar sobre los niveles de adopción de la educación a distancia de los profesores, es fantástico que la mayoría de los profesores estén preparados para el aprendizaje a distancia cuando se haya implementado la política de trabajo desde casa. Sorprendentemente, una quinta parte de los profesores son innovadores y solo hay unos pocos profesores que están rezagados. Hasta el momento los docentes son ágiles en la adopción de la educación a distancia durante la crisis del COVID-19. Aunque es necesario seguir investigando para aclarar si los profesores adoptan y practican el aprendizaje a distancia apropiadamente o viceversa, este hecho es una señal optimista para adaptar y adoptar de manera efectiva la instrucción remota a los entornos escolares formales en el futuro.

\section{Conclusiones}

El presente estudio ha dado una descripción, discusión y reflexión sobre la integración tecnológica de los profesores y adopción del aprendizaje a distancia después de que se haya implementado la política de trabajo desde casa como medida de precaución hacia una mayor transmisión del COVID-19 en Perú. Este estudio se propuso investigar la tecnología utilizada por los profesores como herramientas para el aprendizaje a distancia, el proceso de aprendizaje a distancia realizado por los profesores, y los niveles de adopción del aprendizaje a distancia de los profesores. Los resultados de esta investigación revelan que los profesores tienden a utilizar dispositivos y aplicaciones para instrucción remota que ya usaban en la vida diaria, y aplicaciones globales que tienen más probabilidad que sean elegidos por los profesores para gestionar las aulas virtuales. La mayoría de los profesores se han preparado de inmediato para el aprendizaje a distancia cuando el trabajo desde casa se ha implementado una política para que puedan ser categorizados como los primeros en adoptar la educación a distancia.

La evidencia de este estudio sugiere que confiar en los profesores para llevar a cabo el aprendizaje a distancia con tecnologías con las que están familiarizados es esencial durante el momento crítico. Además, las tendencias identificadas en este estudio deben tenerse en cuenta seriamente para trazar una política de integración tecnológica adecuada para el aprendizaje a distancia en entornos escolares. Una de las ideas podría ser el desarrollo profesional basado en TPACK en varios modos de programas de educación del profesorado (Rahmadi et al., 2020). La agilidad de los docentes en la adopción de la educación a distancia durante la crisis del COVID-19 plantea una señal optimista para adaptarse y adoptar eficazmente la instrucción remota a los entornos escolares formales en el futuro.

Los hallazgos empíricos de este estudio proporcionan una nueva comprensión de la integración tecnológica de los profesores y la adopción del aprendizaje a distancia en medio de los impactos globales sin precedentes del COVID-19. Sin embargo, el estudio actual se ha centrado en examinar la integración tecnológica de los profesores y la adopción del aprendizaje a distancia en un país, independientemente de las materias de enseñanza y los niveles escolares. 


\section{Referencias}

Adnan, M., Kalelioglu, F., \& Gulbahar, Y. (2017). Assessment of a multinational online faculty development program on online teaching: Reflections of candidate etutors. Turkish Online Journal of Distance Education, 18(1), 22-38.

Aldunate, R., \& Nussbaum, M. (2013). Teacher adoption of technology. Computers in Human Behavior, 29(3), 519-524.

Anderson, L. W., \& Bloom, B. S. (2001). A taxonomy for learning, teaching, and assessing: A revision of Bloom's taxonomy of educational objectives. Longman.

Anderson, T., \& Dron, J. (2017). Integrating learning management and social networking systems. Italian Journal of Educational Technology, 25(3), 5-19.

Annamalai, N. (2019). Using WhatsApp to extend learning in a blended classroom environment. Teaching English with Technology, 19(1), 3-20.

Cetinkaya, L., \& Sütçü, S. S. (2018). The effects of Facebook and WhatsApp on success in English vocabulary instruction. Journal of Computer Assisted Learning, 34(5), 504-514.

Clausen, J. M., Bunte, B., \& Robertson, E. T. (2020). Professional development to improve communication and reduce the homework gap in grades 7-12 during COVID-19 transition to remote learning. Journal of Technology and Teacher Education, 28(2), 443-451.

Corno, L. (2000). Looking at homework differently. The Elementary School Journal, $100(5), 529-548$.

Corno, L., \& Xu, J. (2004). Homework as the job of childhood. Theory into practice, 43(3), 227-233.

Davey, B. (1988). How do classroom teachers use their textbooks? Journal of Reading, 31(4), 340-345.

Davies, R. S., \& West, R. E. (2014). Technology integration in schools. En Handbook of Research on Educational Communications and Technology (págs. 841-853). New York: Springer.

Doucet, A., Netolicky, D., Timmers, K., \& Tuscano, F. J. (2020). Thinking about pedagogy in an unfolding pandemic: an independent report on approaches to distance learning during COVID19 school closures.

Hall, B. H., \& Khan, B. (2003). Adoption of new technology (No. w9730), National bureau of economic research.

Heggart, K. R., \& Yoo, J. (2018). Getting the most from google classroom: A pedagogical framework for tertiary educators. Australian Journal of Teacher Education, 43(3), 9.

Holcomb, Z. C. (2016). Fundamentals of descriptive statistics. Routledge.

Innovations in open and flexible education. (2018). En H. K. Singh, T. M. Lim, T. K. Woo, \& M. Fadzil, Mobile learning support to distance learners: using WhatsApp Messenger (págs. 109-119). Singapore: Springer.

Koehler, M. J., \& Mishra, P. (2005). What happens when teachers design educational technology? The development of technological pedagogical content knowledge. Journal of educational computing research, 32(2), 131-152. 
Kotrlik, J. W., \& Redmann, D. H. (2009). Technology adoption for use in instruction by secondary technology education teachers. Journal of Technology Education, 21(1), 44-59.

Lee, J. H. (2012). Cooperative Design,Visualization, and Engineering. En Real-Time Mobile Distance Learning System for Smartphone (págs. 24-32). Berlin: Springer.

Levin, K. A. (2006). Study design III: Cross-sectional studies. Evidence-based dentistry, $7(1), 24-25$.

Martinez-Cola, M., English, R., Min, J., Peraza, J., Tambah, J., \& Yebuah, C. (2018). When pedagogy is painful: Teaching in tumultuous times. Teaching Sociology, 46(2), 97-111.

McDonald, C. V. (2016). Evaluating junior secondary science textbook usage in Australian schools. Research in Science Education, 46(4), 481-509.

Mishra, P., \& Koehler, M. J. (2006). Technological pedagogical content knowledge: A framework for teacher knowledge. Teachers college record, 108(6), 1017-1054.

Nawaila, M. B., \& Bicen, H. (2018). WhatsApp as a tool for distance learning. Ponte Journal, 74(1).

Ouadoud, M., Nejjari, A., Chkouri, M. Y., \& El-Kadiri, K. E. (2017). Proceedings of the Mediterranean Symposium on Smart City Applications. En Learning management system and the underlying learning theories (págs. 732-744). Cham: Springer.

Rahmadi, I. F., Hayati, E., \& Nursyifa, A. (2020). Comparing Pre-Service Civic Education Teachers' TPACK Confidence across Course Modes: Insights for Future Teacher Education Programs. Research in Social Sciences and Technology, 5(2), 113-133.

Redmann, D., \& Kotrlik, J. (2009). Family and consumer sciences teachers' adoption of technology for use in secondary classrooms. Journal of Family and Consumer Sciences, 27(1), 29-45.

Rogers, E. M. (1962). Diffusion of innovations. New York: Free Press of Glencoe.

Rozak, A., \& Albantani, A. M. (2018). Desain perkuliahan bahasa arab melalui google classroom. Arabiyat: Jurnal Pendidikan Bahasa Arab dan Kebahasaaraban, 5(1), 83-102.

Safei, S., Amin, M. A., Rose, A. N., \& Rahman, M. N. (2011). Instant e-teaching framework model for live online teaching. International Journal of Computer Science Issues, 8(2), 84-91.

Saleh, S. T. (2019). Chat and Learn: Effectiveness of Using WhatsApp as a Pedagogical Tool to Enhance EFL Learners Reading and Writing Skills. International Journal of English Language and Literature Studies, 8(2), 61-68.

Sepasgozar, S. M., \& Davis, S. (2018). Construction technology adoption cube: An investigation on process, factors, barriers, drivers and decision makers using NVivo and AHP analysis. Buildings, 8(6), 74.

Shin, D. H., Shin, Y. J., Choo, H., \& Beom, K. (2011). Smartphones as smart pedagogical tools: Implications for smartphones as u-learning devices. Computers in Human Behavior, 27(6), 2207-2214.

Singh, H. K., Lim, T. M., Woo, T. K., \& Fadzil, M. (2018). Innovations in open and flexible education. En Mobile learning support to distance learners: using WhatsApp Messenger (págs. 109-119). Singapore: Springer. 
Revista de Tecnología de Información y Comunicación en Educación • Volumen 15, Nㅜ 1. Enero-abril 2021

Straub, E. T. (2009). Understanding technology adoption: Theory and future directions for informal learning. Review of Educational Research, 79(2), 625-649.

Suldo, S. M., Shaunessy, E., \& Hardesty, R. (2008). Relationships among stress, coping, and mental health in high-achieving high school students. Psychology in the Schools, 45(4), 273-290.

Tondeur, J., Devos, G., Van Houtte, M., van Braak, J., \& Valcke, M. (2009). Understanding structural and cultural school characteristics in relation to educational change: the case of ICT integration. Educational studies, 35(2), 223235.

Tuncay, N. (2016). Smartphones as Tools for Distance Education. Online Submission, 6(2), 20-30.

Vázquez-Cano, E. (2014). Mobile distance learning with smartphones and apps in higher education. Educational Sciences: Theory and Practice, 14(4), 1505-1520.

Walker, D. S., Lindner, J. R., Murphrey, T. P., \& Dooley, K. (2016). Learning management system usage. Quarterly Review of Distance Education, 17(2), 4150.

Wang, X., \& Cheng, Z. (2020). Cross-Sectional Studies. Chest, 158(1), S65-S71.

$\mathrm{Xu}$, J. (2011). Homework Completion at the Secondary School Level: A Multilevel Analysis. The Journal of Educational research, 104(3), 171-182.

Xu, J., \& Yuan, R. (2003). Doing homework: Listening to students,'parents,'and teachers' voices in one urban middle school community. School Community Journal, 13(2), 25. 